Impact of COVID-19 in pediatric oncology care in Latin America during the first year of the pandemic

Gabriela Villanueva ${ }^{1}$, Claudia Sampor ${ }^{2}$, Julia Palma ${ }^{3}$, Milena Villarroel $^{3}$, Diana Valencia ${ }^{4}$, Mercedes Garcia Lombardi ${ }^{5}$, Wendy Gómez García ${ }^{6}$, Eva Lezcano Caceres ${ }^{7}$, Victoria Sobrero $^{8}$, Lilia Garcia Rodriguez ${ }^{9}$, Victor Cabrera ${ }^{10}$, Ivan Maza ${ }^{11}$, Thelma Velasquez ${ }^{12}$, Cecilia Ugaz ${ }^{13}$, Jacqueline Montoya ${ }^{13}$, Rosdali Diaz Coronado ${ }^{13}$, Natalia Gonzalez ${ }^{14}$, Simone Aguiar ${ }^{15}$, Agustín Dabezies ${ }^{16}$, Florencia Moreno ${ }^{17}$, Susan Sardinas ${ }^{18}$, Yessika Gamboa $^{19}$, ESSY MARADIEGUE ${ }^{13}$, Ligia $\mathrm{Fu}^{20}$, Pascale Gassant ${ }^{21}$, Katiuska Moreno ${ }^{22}$, Oscar Gonzalez-Ramella ${ }^{23}$, Magdalena Schelotto ${ }^{24}$, Sandra Luna-Fineman ${ }^{25}$, Celia Antoneli $^{26}$, Soad Fuentes Alabi ${ }^{27}$, Silvana Luciani ${ }^{28}$, Andrea Cappellano ${ }^{29}$, Guillermo Chantada $^{1}$, and Liliana Vasquez ${ }^{28}$

${ }^{1}$ Austral University Hospital

${ }^{2}$ Hospital de Pediatría "Prof Dr JPGarrahan"

${ }^{3}$ Hospital Luis Calvo Mackenna

${ }^{4}$ IMAT Oncomédica

${ }^{5}$ Ricardo Gutiérrez Children's Hospital

${ }^{6}$ Robert Reid Cabral Children's Hospital

${ }^{7}$ Hospital Central Instituto de Prevision Social

${ }^{8}$ Hospital Zonal Juan Ramon Carrillo

${ }^{9}$ Centro Universitario contra el Cáncer UANL

${ }^{10}$ Hospital Regional de Rio Blanco

${ }^{11}$ Hospital Nacional Edgardo Rebagliati Martins

${ }^{12}$ Unidad Nacional de Oncologia Pediatrica

${ }^{13}$ Instituto Nacional de Enfermedades Neoplasicas

${ }^{14}$ Hospital Militar Central

${ }^{15}$ Hospital Santa Marcelina

${ }^{16}$ Pereira Rossell

${ }^{17}$ Registro Onco-pediatrico Hospitalario Argentino (ROHA, Hospital-based Pediatric Cancer Registry from Argentina), Instituto Nacional del Cáncer

${ }^{18}$ Hospital del Niño Dr. Ovidio Aliaga Uría

${ }^{19}$ Hospital Nacional de Niños

${ }^{20}$ Hospital Escuela Universitario

${ }^{21}$ Hôpital Saint - Damien

${ }^{22}$ Hospital Verdi Cevallos Balda - Hospital especialidades Portoviejo

${ }^{23}$ Hospital Civil de Guadalajara Dr. Juan I. Menchaca

${ }^{24}$ Pereira Rossell Hospital Center

${ }^{25}$ Children's Hospital Colorado

${ }^{26}$ Universidade Nove the Julhio

${ }^{27}$ Benjamin Bloom Hospital 
${ }^{28}$ Pan American Health Organization

${ }^{29}$ Instituto de Oncologia Pediátrica - IOP/GRAACC/UNIFESP

January 28, 2022

\begin{abstract}
The ongoing COVID-19 pandemic strained medical systems worldwide. We report on the impact on pediatric oncology care in Latin American (LATAM) during its first year. Four cross-sectional surveys were electronically distributed among pediatric onco-hematologist in April/June/October 2020, and April/2021 through the Latin American Society of Pediatric Oncology (SLAOP) email list and St Jude Global regional partners. 453 pediatric onco-hematologists from 20 countries responded the first survey with subsequent surveys response rates above $85 \%$. More than $95 \%$ of participants reported that treatment continued without interruption for new and active on-going patients, though with disruptions in treatment availability. During the first three surveys, respondents reported suspensions of outpatient procedures (54.2\%), a decrease in oncologic surgeries (43.6\%), radiotherapy (28.4\%), stem cell transplants (SCT) (69.3\%), and surveillance consultations (81.2\%). Logistic regression analysis showed that at the beginning of the first wave, participants from countries with healthcare expenditure below $7 \%$ were more likely to report a decrease in outpatient procedures (OR:1.84, 95\%C:1.19;2.8), surgeries (OR:3, 95\%CI:1.9;4.6) and radiotherapy (OR:6, 95\%CI:3.5;10.4). Suspension of surveillance consultations was higher in countries with COVID-19 case fatality rates above $2 \%$ (OR:3, 95\%CI:1.4;6.2) and SCT suspensions in countries with COVID-19 incidence rate above 100 cases per 100,000 (OR:3.48, 95\%CI:1.6;7.45). Paradoxically, at the beginning of the second wave with COVID-19 cases rising exponentially, most participants reported improvements in cancer services availability. Our data show the medium-term collateral effects of the pandemic on pediatric oncology care in LATAM, which might help delineate oncology care delivery amid current and future challenges posed by the pandemic.
\end{abstract}

\title{
Impact of COVID-19 in pediatric oncology care in Latin America during the first year of the pandemic
}

Authors: Gabriela Villanueva ${ }^{1}$, Claudia Sampor ${ }^{2}$, Julia Palma ${ }^{3}$, Milena Villarroel ${ }^{3}$, Diana Valencia ${ }^{4}$, Mercedes García Lombardi ${ }^{5}$, Wendy Gomez Garcia ${ }^{6}$, Eva Lezcano Caceres ${ }^{7}$, Victoria Sobrero ${ }^{8}$, Lilia Garcia ${ }^{9}$, Victor Cabrera ${ }^{10}$, Ivan Maza ${ }^{11}$, Thelma Velasquez ${ }^{12}$, Cecilia Ugaz ${ }^{13}$, Jacqueline Montoya Vasquez ${ }^{13}$, Rosdali Diaz Coronado ${ }^{13}$, Natalia Gonzalez ${ }^{14}$, Simone Aguiar ${ }^{15}$, Agustin Dabezies ${ }^{16}$, Florencia Moreno ${ }^{17}$, Susan Sardinas $^{18}$, Yessika Gamboa ${ }^{19}$, Essy Maradiegue ${ }^{20}$, Ligia $\mathrm{Fu}^{21}$, Pascale Gassant ${ }^{22}$, Katiuska Moreno ${ }^{23}$, Oscar Gonzales ${ }^{24}$, Magdalena Schelotto ${ }^{25}$, Sandra Luna-Fineman ${ }^{26}$, Celia Gianotti Antoneli ${ }^{27}$, Soad FuentesAlabi $^{28,29}$, Silvana Luciani ${ }^{28}$, Andrea Cappellano ${ }^{30}$, Guillermo Chantada ${ }^{1,25}$, Liliana Vasquez ${ }^{28,31}$

${ }^{1}$ Hospital Austral, Pediatrics Hematology and Oncology, Pilar, Argentina,

${ }^{2}$ Hospital J.P Garrahan, Service of Hematology/Oncology, Buenos Aires, Argentina

${ }^{3}$ Hospital Luis Calvo Mackenna, Pediatric Oncology, Santiago, Chile

${ }^{4}$ Hospital Universitario de Santander IMAT oncomedica, Pediatric Oncology, Colombia

${ }^{5}$ Hospital de Niños Ricardo Gutierrez, Pediatric Oncology, Buenos Aires, Argentina

${ }^{6}$ Dr. Robert Reid Cabral Children's Hospital, Hematology-Oncology, Dominican Republic

${ }^{7}$ Hospital Central Instituto de Prevision Social, Pediatric Oncology, Asunción, Paraguay

${ }^{8}$ Hospital Ramon Carrillo, Pediatric Oncology, San Carlos De Bariloche, Argentina

${ }^{9}$ Centro Universitario Contra el Cáncer UANL

${ }^{10}$ Hospital Regional Río Blanco, Pediatric Oncology, Orizaba, Mexico

${ }^{11}$ Hospital Rebagliati, Pediatric Oncology, Lima, Peru 
12 Unidad Nacional de Oncología Pediátrica, Pediatric Oncology, Guatemala City, Guatemala

13 Instituto Nacional de Enfermedades Neoplásicas, Lima, Peru

${ }^{14}$ Hospital Militar Nacional de Colombia, Bogotá, Colombia

${ }^{15}$ Hospital Sta Marcelina/ TUCCA, Pediatric Oncology, São Paulo, Brazil

${ }^{16}$ Pediatric Hemato Oncology Honorary Advisor Consultant MUCAM, Montevideo, Uruguay

17 Registro Onco-pediatrico Hospitalario Argentino (ROHA, Hospitalbased Pediatric Cancer Registry from Argentina), Registry, Instituto Nacional del Cancer, Argentina

18 Hospital del Niño Ovidio Aliaga Uria, La Paz, Bolivia

19 Hospital Nacional de Niños, Pediatric Oncology, San José, Costa Rica

20 Ministry of health, Prevention of cancer directorate, Lima, Peru

21 Hospital Escuela Universitario, Hemato-Oncologia, Tegucigalpa, Honduras,

${ }^{22}$ Hôpital Saint - Damien, Pediatric Oncology, Port-au-Prince, Haiti

${ }^{23}$ Hospital Verdi Cevallos Balda - Hospital especialidades Portoviejo, Docente de pregrado de hematologia universidad laica Eloy Alfaro de Manabi, Manabi, Ecuador

24 Hospital Civil de Guadalajara Dr. Juan I Menchaca. Guadalajara Jalisco México

25 Hospital Pereira Rossell, Pediatric Oncology, Montevideo, Uruguay

${ }^{26}$ University of Colorado School of Medicine, Aurora, CO 80045, USA; Department for the Management of Noncommunicable Diseases, Disability, Violence and Injury Prevention, World Health Organization Geneva, Switzerland

27 Universidade Nove the Julhio, São Pablo, Brazil

28 Pan American Health Organization, Non-Communicable Diseases, Washington D.C., USA

${ }^{29}$ Hospital Nacional de Niños Benjamin Bloom y Fundacion Ayudame a Vivir Pro- Ninos con Cancer de El Salvador, Pediatric Oncology, San Salvador, El Salvador

30 Instituto de Oncologia Pediátrica - IOP/GRAACC/UNIFESP, Department of Pediatric Neuro-oncology, São Paulo, Brazil

31 Universidad de San Martín de Porres, Facultad de Medicina, Centro de Investigación de Medicina de Precisión. Lima, Perú

Word count abstract : 250

Word count main text: 3098

Supporting Information files : 1

Number of tables : 1

Number of figures : 4

Running title : Covid-19 impact pediatric cancer care in LATAM

Keywords: Pediatric cancer, COVID-19, healthcare delivery, survey, Latin America, low-and-middle-income countries.

Table of abbreviations 


\begin{tabular}{ll}
\hline LATAM & Latin America \\
\hline LMIC & Low-and-middle-income countries \\
SLAOP & Latin American Society of Pediatric Oncology \\
SCT & Stem cell transplant \\
HCE & Healthcare expenditure \\
OR & Odds ratio \\
COVID-19 & Coronavirus 2019 disease \\
SARS & Severe acute respiratory virus \\
PPE & Personal protective equipment \\
HIC & High income countries \\
PAHO & Pan American Health Organization \\
GDP & Gross domestic product \\
NCDs & Non-communicable diseases \\
\hline
\end{tabular}

\begin{abstract}
Background: The ongoing COVID-19 pandemic strained medical systems worldwide. We report on the impact on pediatric oncology care in Latin American (LATAM) during its first year.

Method : Four cross-sectional surveys were electronically distributed among pediatric onco-hematologist in April/June/October 2020, and April/2021 through the Latin American Society of Pediatric Oncology (SLAOP) email list and St Jude Global regional partners.

Results : 453 pediatric onco-hematologists from 20 countries responded the first survey with subsequent surveys response rates above $85 \%$. More than $95 \%$ of participants reported that treatment continued without interruption for new and active on-going patients, though with disruptions in treatment availability. During the first three surveys, respondents reported suspensions of outpatient procedures $(54.2 \%)$, a decrease in oncologic surgeries (43.6\%), radiotherapy (28.4\%), stem cell transplants (SCT) (69.3\%), and surveillance consultations (81.2\%). Logistic regression analysis showed that at the beginning of the first wave, participants from countries with healthcare expenditure below $7 \%$ were more likely to report a decrease in outpatient procedures (OR:1.84, 95\% C:1.19;2.8), surgeries (OR:3, 95\%CI:1.9;4.6) and radiotherapy (OR:6, $95 \%$ CI:3.5;10.4). Suspension of surveillance consultations was higher in countries with COVID-19 case fatality rates above $2 \%$ (OR:3, 95\%CI:1.4;6.2) and SCT suspensions in countries with COVID-19 incidence rate above 100 cases per 100,000 (OR:3.48, 95\%CI:1.6;7.45). Paradoxically, at the beginning of the second wave with COVID-19 cases rising exponentially, most participants reported improvements in cancer services availability.
\end{abstract}

Conclusion : Our data show the medium-term collateral effects of the pandemic on pediatric oncology care in LATAM, which might help delineate oncology care delivery amid current and future challenges posed by the pandemic.

\title{
Introduction :
}

Severe acute respiratory virus (SARS-CoV-2) outbreak resulting in Coronavirus 2019 disease (COVID-19) caused an unprecedented pandemic that led to more than 200 million cases and 4 million deaths around the world (date August 5, 2021). ${ }^{1}$ One year into the pandemic, multiple measures were implemented by governments attempting to slow the spread of COVID-19 and to ameliorate the burden on their medical systems. ${ }^{2,3}$ These included home isolations, closing borders, schools, nonessential business, and restricting travel within and between countries. The fear generated by the pandemic resulted in patients being unable or unwilling to seek medical care. ${ }^{4}$ The Pandemic exposed unprepared health care systems with limited personal protective equipment (PPE), limited ICU staff and ventilators which affected unprotected vulnerable populations. ${ }^{5}$ Initial cases of COVID-19 occurred in Latin America weeks after Europe and North America struggled with a steep first wave. This allowed Latin American countries to implement early epidemiological 
actions and pre-emptively restrict their medical system's nonessential use. Now, more than a year into the pandemic, the resulting economic and social impact are still unfolding. The development of multiple effective vaccines has provided some relief, though still only $24.2 \%$ of the world population is fully vaccinated, mainly in $\mathrm{HIC}^{6}$, leaving most of the world exposed to new waves of COVID-19, with an increased risk for the development of highly contagious SARS-CoV2 variants.

The survival of pediatric oncology patients depends significantly on a functional medical system that allows for early diagnosis and referrals to tertiary care centers, timely initiation, coordination of care among multidisciplinary teams, and access to supportive care. ${ }^{7}$ Early studies showed that complications and mortality from COVID-19 were lower in pediatric patients compared to adults, ${ }^{8}$ and that pediatric oncology patients were not at elevated risk of poor outcomes as adults cancer patients. ${ }^{9-11}$ However, a recent global study reporting the outcome of pediatric oncologic patients infected with COVID-19 from April 2020 to February 2021, showed that one-fifth of that population had a severe critical infection, and $3.8 \%$ died due to COVID-19, which is four times the mortality reported in the pediatric general population. ${ }^{12}$

A first report on the pandemic's impact on pediatric cancer care delivery in Latin America was published in April 2020 (before the first wave in the region). This study revealed that healthcare expenditure (HCE) was closely associated with higher proportions of disrupted pediatric oncologic treatments. ${ }^{13}$ However, as the pandemic evolved, Latin America became one of the world's hot spots, accounting for more than 30 million cases and more than 1 million deaths. This study provides evidence from four cross-sectional surveys conducted throughout the first year of the pandemic in Latin America, aiming to show how pediatric cancer services adapted to several waves of SARS-CoV2 infection and the disruptions of pediatric oncologic care delivery that resulted from them.

Methods

\section{Study design and participants}

We conducted four cross-sectional surveys of pediatric onco-hematologists throughout twenty Latin American countries during the first year of the COVID-19 pandemic. The participating countries include Argentina, Bolivia, Brazil, Chile, Colombia, Costa Rica, Cuba, Dominican Republic, Ecuador, El Salvador, Guatemala, Haiti, Honduras, Mexico, Nicaragua, Panama, Paraguay, Peru, Uruguay, and Venezuela. The results of the first survey, performed in April 2020, have already been published ${ }^{13}$. The survey was electronically distributed through the Latin American Society of Pediatric Oncology (SLAOP) (the International Society of Pediatric Oncology -SIOP-continental branch) email list in collaboration with St Jude Global and the Pan American Health Organization (PAHO). For each country participating in the study, the national delegate from the SLAOP network was assigned to promote the survey among physicians. The distribution of the online forms included mailshots (through the email list of the SLAOP and the Central and South America Region Branch of St. Jude Global-CASA).

Surveys were carried out at four strategic moments during the pandemic. The first one took place from April 12 to 19, 2020, during the first wave of infections in Europe and North America, but still few cases in LATAM. The second one took place from June 10 to 17, 2020, when cases were rising during the first wave in most LATAM countries. The third one was conducted from October 19 to 26, 2020, when the first wave started to resolve, and, finally, the fourth survey occurred from April 12 to 26, 2021, halfway through the region's second wave.

\section{Survey questionnaires}

Each survey collected data on the individual perspectives of physicians (pediatric oncologist, medical directors, residents, and fellows) working in pediatric onco-hematology departments of public and private health institutions. Other health care providers, such as nurses, psychologists, and physical, occupational and speech therapists, among others were excluded. Each survey contained between 20 and 32 items and was developed to capture data about the disruption and adaptation of pediatric cancer care at a specific time point, aiming to detect changes, as the pandemic evolved. 
Questions on the following topics were included: treatment suspension of newly diagnosed patients and active ongoing cases, chemotherapy protocol modifications, displacement or reduction of medical staff, suspension of outpatient procedures, cancer surgeries, radiation therapy sessions, bone marrow transplantation, and palliative care assessments and care. In addition, some questions were formulated to capture information about strategies implemented to provide continuity of care (telemedicine) and personal perspectives on the greatest impacts of the pandemic on patients' care (delays in diagnosis, treatment abandonment, and outof-pocket expenses).

Health indicators and epidemiologic parameters of COVID-19, such as incidence rate, case fatality rate (number of COVID-19 deaths divided by the total number of cases, multiplied by 100) and healthcare expenditure as a percentage of GDP (HCE) from each country were analyzed for potential association with the impact of treatment of children with cancer. All responses were anonymous and institutional ethical review was not required. After data collection, each country delegate verified the authenticity and consistency of the data provided.

\section{Statistical analysis}

Development indicators from each participating country were obtained from the World Bank Open Data platform $^{6}$. Data on COVID-19 incidence and mortality beginning on February 28, 2020 (first COVID-19 case diagnosed in LATAM) to April 30, 2021 (day the last survey closed) was extracted from the data repository for the 2019 Novel Coronavirus Visual Dashboard operated by the Johns Hopkins University Center for Systems Science and Engineering (JHU CSSE). ${ }^{14}$

Respondent characteristics were reported using descriptive statistics. Categorical variables were assessed by chi-square test. Univariate and multivariable logistic analysis was done for the following health care factors: treatment of new patients, treatment of active/ongoing cases, suspension of outpatient procedures, suspension of oncologic surgeries, suspension of radiation therapy sessions, suspension of stem cell transplant (SCT) and suspension of surveillance consultations. We calculated crude odds ratios and variables associated with significant impact in a multivariable logistic regression analysis.

Radiation therapy sessions and SCT proportions were calculated based on the total number of participants that reported having access to radiation therapy or SCT. Participants who reported no access to those services or answered "Do not know" were excluded. Significance was established when p values were less than 0.05. Statistical analyses were performed using RStudio version 1.2.1335.

\section{Results:}

From 795 pediatric oncologists, medical directors and residents/fellows contacted, the response rate among all four surveys ranged between 43-57\%. A total of 453 of 795 pediatric onco-hematologists from 20 countries responded to the first survey. The participation for subsequent surveys remained above $85 \%$ among the original respondents: 408 participants in June 2020 (90\%), 386 in October 2020 (85.2\%) and 389 in April 2021 (85.9\%). (Supplementary data Table S1). In all four surveys, respondents were pediatric oncologist (50-59\%), medical directors (31\%) and residents/fellows (10\%). Participants were affiliated to public hospitals (58-63\%), public-private $(22-24 \%)$ or private (15-17\%). The most frequent facility type was children's hospitals (41\%), followed by general hospital (35\%) and cancer centers (23\%).

The first three surveys took place during the beginning, peak and resolution of the first wave in most participating countries, except for Uruguay, whose first wave started late in April 2021 (Figure 2 and 3). During those first three surveys, on average, respondents reported partial or complete suspension of outpatient procedures $(54.2 \%)$, decreases in surgical services (43.6\%), radiation therapy sessions (28.4\%), SCT (69.3\%) and off therapy surveillance consultations (81.2\%) (Table 1). Even though more than $95 \%$ of the participants surveyed reported that new and active patients received oncologic treatment during all four surveys, they also reported disruptions in treatment availability that conditioned the type of treatment they offered (Figure 1, 2 and 3$)$.

Univariate logistic regression analysis was performed on each survey. Factors including incidence rate of 
more than 100 cases per 100,000, case fatality rate above $2 \%$ and HCE less than $7 \%$ were significantly associated with suspensions of some or all of the following services: outpatient procedures, oncologic surgeries, patient surveillance, radiotherapy sessions and SCT. In contrast, suspension of treatment of new and active ongoing patients was not, as physicians continue treating patients with the treatments modalities that were available. Multivariable logistic regression analysis showed that when COVID-19 cases were rising at the beginning of the first wave (June 2020), LATAM countries with an HCE below 7\% were more likely to report a decrease in outpatient procedures (OR:1.84, 95\%CI: 1.19;2.8) and surgeries (OR:3, 95\%CI: 1.9;4.6), controlling for other indicators of COVID-19 burden. In addition, suspension of radiation therapy sessions also increased in those countries with HCE less than 7\% (OR:6, 95\%CI: 3.5;10.44). Surveillance consultations were suspended in countries with a case fatality rate above $2 \%$ (OR:3, 95\%CI:1.4;6.2); and SCT were suspended in countries where COVID-19 incidence rate was above 100 cases per 100,000 (OR: 3.48, 95\%CI: 1.6;7.45) (Supplementary data Table S4). At the end of the first wave, in October 2020, the association between HCE and the suspension of outpatient procedures, surgeries, radiotherapy sessions and SCT suspensions remained, even as the first wave resolved and indicators of national burden of COVID-19 improved in most countries (Supplementary data Table S5).

In April 2021 (beginning of the second wave), with cases of COVID-19 rising exponentially again in most countries, most participants reported an improvement in pediatric cancer services availability. Only $36 \%$ of respondents reported suspension of outpatient procedures (down from 50\% in June 2020), 30.6\% reported suspensions in surgeries (down from $41 \%$ in June 2020), $16 \%$ suspension of radiotherapy sessions (down from $25.1 \%$ in June 2020), 37.5\% suspension of SCT (down from $68.1 \%$ in June 2020); and 48.1\% reported suspension of surveillance consultations (down from $80.7 \%$ in June 2020) (Table 1). In April 2021, only countries with HCE below $7 \%$ continued to have an increase in radiotherapy sessions suspension (OR:3.16, 95\%CI:1.7;5.89) and in SCT suspensions (OR:7.16 - 95\%CI:3.17;16.15), while other indicators of national COVID-19 burden did not show any association (Supplementary data Table S6).

At the beginning of the pandemic, $35.6 \%$ of participants reported chemotherapy regimen modifications due to drug shortages and $45.2 \%$ a significant decrease in access to blood products. Deeper into the pandemic, (June-October 2020), chemotherapy availability improved as only $15 \%$ and $18 \%$ of participants reported chemotherapy shortages. However, an average of $55 \%$ still reported decrease in access to blood products during the last 3 surveys (Supplementary data Table S7).

As shown in Figure 4, participants reported that since the beginning of the pandemic there were frequent delays in diagnosis (33\%), frequent treatment abandonment (17.2\%) and an increase in family out-of-pocket expenses $(42.2 \%)$.

Responses about access to SCT and radiation therapy varied within countries and between surveys. During all four surveys, on average, $48.5 \%$ of participants reported not having access to SCT and $15.4 \%$ of participant reported not having access to radiation therapy.

Notably, during all four surveys, from $60 \%$ to $82 \%$ of respondents reported some percentage of hospital staff reduction due to COVID-19 infection or quarantine, but only maximum of 3.75\% reported more than a $50 \%$ staff reduction (in April 2020). In addition, a maximum of 1.5\% of respondents reported more than a $50 \%$ displacement of pediatric oncologist to work in other sectors of the hospital due to COVID-19 (in June 2020) (Table1).

On average, $55 \%$ of participants had access to some form of telemedicine during the first year of the pandemic (including institutional telemedicine platforms and non-professional communication channels such as WhatsApp, Facebook or Zoom). In April 2021, more than $80 \%$ of participants reported that they would like to have an institutional telemedicine platform to follow their patients, as more than half of them (50.4\%) had only access to non-professional communication channels.

By April 2021, 99 (25\%) participants reported having contracted COVID-19, 298 (76\%) reported being immunized with two doses of a SARS-CoV2 vaccine, and $64(16.4 \%)$ with only one dose. Only 5 participants $(1.5 \%)$ refused to be vaccinated (Supplementary data Table S9) 


\section{Discussion:}

The COVID-19 pandemic is an unprecedented global crisis that impacted pediatric cancer health care systems worldwide, with a greater effect on low and middle-income countries (LMIC). ${ }^{2,3,12,15-17}$ One year into the pandemic, the initial fears and concerns have evolved along with treatment and prevention strategies for COVID-19 and multiple effective vaccines have been developed in record time. As the pandemic continues to unfold, our 4 surveys performed over the first year of the pandemic in Latin America show the mediumterm effects on the care of pediatric cancer patients; and reveal how the medical systems and medical professionals adapted to the different health care crises that originated from the pandemic.

Worldwide, the need to adapt to an uncertain future led to rationing of treatment delivery by suspensions of most pediatric cancer services. ${ }^{13,16-18}$ A recent global study reported that $55.8 \%$ of children with cancer, had modifications in their cancer-directed therapies during the pandemic. In India, a group reported $36.1 \%$ of 1146 pediatric patients with cancer experienced treatment delays after a country-wide lockdown. In alignment with these findings, our data shows different levels of disruption of cancer services, such as suspension of SCT, radiotherapy sessions, oncologic surgeries, and chemotherapy modifications throughout the year. Specifically, from April 2020 to October 2020, most countries experienced the first wave with intense constraints imposed by government lockdowns. Therefore, the answers to our surveys reflect cautionary adaptation, as most medical systems organized to face unpredictable health care delivery crises. Not surprisingly, higher levels of treatment disruptions were reported in countries with HCE below 7\%, and in countries with higher national burden of COVID-19, reflected by a higher incidence rate and case fatality rate above 2\%. However, by April 2021, amid the second wave in the region, the respondents' answers reflected a better understanding of the effect of COVID-19 on children with cancer, a better preparedness of the medical system to safely deliver cancer care and the resilience of pediatric cancer professionals in the region, who were able to re-institute most cancer care services, in the midst of the pandemic.

A recent questionnaire led by the World Health Organization on the impact of COVID-19 on noncommunicable diseases (NCDs) resources and services showed that $66 \%$ of participating countries (mainly upper-middle and high-income countries). included NCDs services in the list of national essential health services ${ }^{19}$. However, in LMIC, access to quality cancer care was limited even before the pandemic, as health care systems struggled with strained resources. ${ }^{20-22}$ The COVID-19 pandemic exacerbated the scarcity of these resources; and institutions reported decreases in financial support, as well as increased dependency on social organizations to provide help in transportation and family accommodation during treatments. ${ }^{17}$ By April 2021, almost half of the participants in our surveys reported patients' families experiencing increased economic burden of cancer care, including out-of-pocket expenses. This highlights the overall increased social impact of the pandemic on cancer therapy delivery and financing.

Delayed diagnosis and treatment abandonment are well-described problem in LMIC. ${ }^{21}$ Early in the pandemic, different studies reported a decrease of newly diagnosed children with cancer, delays in referral to tertiary centers ${ }^{7,9,22,23}$ and increased treatment abandonment. ${ }^{7,9,15,22,23}$ However, by April 2021, our data show that even though one third of respondents reported persistent delays in diagnosis, only $17.3 \%$ reported treatment abandonment as a persistent problem, which might be related to an increase in the use of telemedicine as a modality to assure continuity of care. Telemedicine (including video, web and telephone-based interventions) had been implemented pre-pandemic in malignant hematologic patients; and proved to be a feasible and acceptable form of intervention. ${ }^{24}$ The changing landscape of health care delivery created by the pandemic opened the opportunity to implement telemedicine to provide continuity of care to pediatric oncology patients ${ }^{25,26}$. In our data, on average during all four surveys, close to half of the participants reported not having access to telemedicine. In addition, by April 2021, 50.4\% of participants reported only having access to non-professional communication channels, such as WhatsApp, Facebook or Zoom, to provide continuity of care. Even though those communication channels worked in areas with poor internet connection, $84 \%$ of respondents reported interest in having a formal telemedicine platform to manage and follow patients.

With multiple and effective vaccines developed, vaccine hesitancy, even among health care professionals is one of the biggest challenges to control the pandemic. ${ }^{27}$ In Latin America, vaccine rollouts started with 
different levels of success, and governments experienced difficulties to deliver doses to their population. By the end of April 2021, vaccination rates in Latin America ranged from 0\% to 35\% for two doses and $0 \%$ to $15 \%$ for one dose (Supplementary data Table S9). ${ }^{28}$ However, our data at that time point revealed that $76.6 \%$ of participants had received two doses of a vaccine, $16.4 \%$ one dose and only $1.5 \%$ refused the vaccine, reflecting a high level of vaccine acceptance among pediatric oncologists in the region.

Our study has several strengths and limitations. One of the biggest strengths of this study is its high and stable participation rate from 20 Latin America countries with pediatric oncology programs. However, responses are subjective, as surveys solicited pediatric oncologists' insights on the impact of the pandemic, and on the barriers to cancer care delivery. There is a disparity in the number of participants from different countries throughout all four surveys and the calculated proportion of answers at each time point is conditioned by the number of participants per country. Therefore, it is difficult to compare countries' data within and between surveys. However, as most Latin America countries shared a similar infection wave pattern during the first year of the pandemic, we believe the information collected is meaningful.

In conclusion, our data shows differences between participants' responses during the first and second waves of COVID-19 in Latin America. During the first wave, treatment disruptions (suspension of SCT, radiotherapy sessions, outpatient procedures, surveillance consultations, and surgeries) were prevalent. In addition, in countries with high COVID-19 burden and HCE below 7\%, pediatric oncologists reported more extreme disruptions at the peak of the first wave. Nonetheless, all countries reported an improvement and recovery of pediatric cancer services during the second wave.

\section{Conflict of interest:}

GC receives fees from Bayer and Elea-Phoenix (Argentina) and participates on the data safety monitoring board or advisory board for Bayer (Larotrectinib).

SLF receives personal fees from Riverboat Study (Research into Visual endpoints and Rb

Health outcomes - 5R01CA225005-03- Salary support), WHO NCD Childhood Cancer - consultant (salary support)-, St Jude Global consultant, St Jude Children's Research Hospital, Memphis TN, USA, Global Retinoblastoma studies (salary Support).

SLF is also a SLAOP Board member and works at the Retinoblastoma Advisory committee, St Jude Global Alliance, St Jude Children's Research Hospital.

All other authors declare no competing interests.

Role of funding source : No funding was used for this study

Ethics committee approval: All responses were anonymous and institutional ethical review was not required.

\section{DATA SHARING:}

The data that support the findings of this study are available from the corresponding author upon reasonable request.

\section{References}

1. World Health Organisation. WHO Coronavirus Disease (COVID-19) Dashboard. Available: https://covid19.who. int. 5th August 2021.

2. Graetz D, Agulnik A, Ranadive R, et al. Global effect of the COVID-19 pandemic on pediatric cancer care: a cross-sectional study. Lancet Child Adolesc Health . 2021;5(5):332-340. doi:10.1016/S2352-4642(21)00031-6

3. Radhakrishnan VS, Nair RKS, Goel G, Ramanan V, Chandy M, Nair R. COVID-19 and haematology services in a cancer centre from a middle-income country: adapting service delivery, balancing the known and unknown during the pandemic. Ecancermedicalscience . 2020;14:1110. doi:10.3332/ecancer.2020.1110 
4. Saab R, Obeid A, Gachi F, et al. Impact of the coronavirus disease 2019 (COVID-19) pandemic on pediatric oncology care in the Middle East, North Africa, and West Asia region: A report from the Pediatric Oncology East and Mediterranean (POEM) group. Cancer . 2020;126(18):4235-4245. doi:10.1002/cncr.33075

5. Molica M, Mazzone C, Cordone I, Pasquale A, Niscola P, de Fabritiis P. SARS-CoV-2 infection anxieties and general population restrictions delay diagnosis and treatment of acute haematological malignancies. $\mathrm{Br}$ J Haematol . 2020;190(1):e5-e8. doi:10.1111/bjh.16785

6. WDI - Home. Accessed August 10, 2021. https://datatopics.worldbank.org/world-developmentindicators/

7. Carai A, Locatelli F, Mastronuzzi A. Delayed referral of pediatric brain tumors during COVID-19 pandemic. Neuro Oncol . 2020;22(12):1884-1886. doi:10.1093/neuonc/noaa159

8. Dong Y, Mo X, Hu Y, et al. Epidemiology of COVID-19 Among Children in China. Pediatrics . 2020;145(6). doi:10.1542/peds.2020-0702

9. Dinmohamed AG, Visser O, Verhoeven RHA, et al. Fewer cancer diagnoses during the COVID-19 epidemic in the Netherlands. Lancet Oncol . 2020;21(6):750-751. doi:10.1016/S1470-2045(20)30265-5

10. Hrusak $\mathrm{O}$, Kalina $\mathrm{T}$, Wolf $\mathrm{J}$, et al. Flash survey on severe acute respiratory syndrome coronavirus-2 infections in pediatric patients on anticancer treatment. Eur J Cancer . 2020;132:11-16. doi:10.1016/j.ejca.2020.03.021

11. Terenziani M, Massimino M, Biassoni V, et al. SARS-CoV-2 disease and children under treatment for cancer. Pediatr Blood Cancer . Published online May 6, 2020:e28346. doi:10.1002/pbc.28346

12. Mukkada S, Bhakta N, Chantada GL, et al. Global characteristics and outcomes of SARS-CoV-2 infection in children and adolescents with cancer (GRCCC): a cohort study. Lancet Oncol . Published online August 26, 2021:S1470-2045(21)00454-X. doi:10.1016/S1470-2045(21)00454-X

13. Vasquez L, Sampor C, Villanueva G, et al. Early impact of the COVID-19 pandemic on pediatric cancer care in Latin America. The Lancet Oncology . 2020;21(6):753-755. doi:10.1016/S1470-2045(20)30280-1

14. Dong E, Du H, Gardner L. An interactive web-based dashboard to track COVID-19 in real time. The Lancet Infectious Diseases . 2020;20(5):533-534. doi:10.1016/S1473-3099(20)30120-1

15. Raza MR, Maqsood S, Rana ZA, et al. Impact of COVID-19 on the Children with Cancer in 6 Pediatric Oncology Units (POU's) of Pakistan-a Multi-Center Study. Cancer Invest . Published online April 18, 2021:1-11. doi:10.1080/07357907.2021.1916027

16. Seth R, Das G, Kaur K, et al. Delivering pediatric oncology services during a COVID-19 pandemic in India. Pediatr Blood Cancer . 2020;67(12):e28519. doi:10.1002/pbc.28519

17. Sharma J, Mahajan A, Bakhshi S, et al. The impact of COVID-19 pandemic on access to treatment for children with cancer in India and treating center practices. Cancer . n/a(n/a). doi:10.1002/cncr.33945

18. Alshahrani M, Elyamany G, Sedick Q, et al. The Impact of COVID-19 Pandemic in Children With Cancer: A Report From Saudi Arabia.Health Serv Insights . 2020;13:1178632920984161. doi:10.1177/1178632920984161

19. The impact of the COVID-19 pandemic on noncommunicable disease resources and services: results of a rapid assessment. Accessed December 15, 2021. https://www.who.int/publications-detailredirect/9789240010291

20. Rodriguez-Galindo C, Friedrich P, Alcasabas P, et al. Toward the Cure of All Children With Cancer Through Collaborative Efforts: Pediatric Oncology As a Global Challenge. J Clin Oncol . 2015;33(27):30653073. doi:10.1200/JCO.2014.60.6376 
21. Rodriguez-Galindo C, Friedrich P, Morrissey L, Frazier L. Global challenges in pediatric oncology. Curr Opin Pediatr . 2013;25(1):3-15. doi:10.1097/MOP.0b013e32835c1cbe

22. Dvori M, Elitzur S, Barg A, et al. Delayed diagnosis and treatment of children with cancer during the COVID-19 pandemic. Int J Clin Oncol . 2021;26(8):1569-1574. doi:10.1007/s10147-021-01971-3

23. Kutluk MT, Ahmed F, Kirazlı M, et al. The effect of the COVID-19 pandemic on pediatric cancer care: lessons learnt from a major pediatric oncology department in Turkey. Ecancermedicalscience . 2021;15:1172. doi:10.3332/ecancer.2021.1172

24. Shah AC, O'Dwyer LC, Badawy SM. Telemedicine in Malignant and Nonmalignant Hematology: Systematic Review of Pediatric and Adult Studies. JMIR Mhealth Uhealth . 2021;9(7):e29619. doi:10.2196/29619

25. Shirke MM, Shaikh SA, Harky A. Implications of Telemedicine in Oncology during the COVID-19 Pandemic. Acta Biomed . 2020;91(3):e2020022. doi:10.23750/abm.v91i3.9849

26. Elkaddoum R, Haddad FG, Eid R, Kourie HR. Telemedicine for cancer patients during COVID-19 pandemic: between threats and opportunities. Future Oncol . 2020;16(18):1225-1227. doi:10.2217/fon-20200324

27. Dror AA, Eisenbach N, Taiber S, et al. Vaccine hesitancy: the next challenge in the fight against COVID-19. Eur J Epidemiol . 2020;35(8):775-779. doi:10.1007/s10654-020-00671-y

28. Ritchie H, Mathieu E, Rodés-Guirao L, et al. Coronavirus Pandemic (COVID-19). Our World in Data . Published online March 5, 2020. Accessed September 17, 2021. https://ourworldindata.org/covidvaccinations

\section{LEGENDS}

Figure 1: Proportion of responses on childhood cancer treatments disruptions (total or partial suspensions) during the first year of COVID-19 pandemic in all participating Latin American countries.

Table 1: Participant responses about treatment disruptions comparing the first wave (April-June-October 2020) and the beginning of second wave in Latin America (April 2021)

Figure 2: South America. Proportion of responses on treatments disruptions (total or partial suspensions) per survey and per country, compared to number of COVID-19 cases/day in each country

Figure 3: Central America. Proportion of responses on treatments disruptions (total or partial suspensions) per survey and per country, compared to number of COVID-19 cases/day in each country.

Figure 4 : Effect of COVID-19 pandemic on treatment abandonment, delays in diagnosis and increase out-of-pocket expenses during the first year of the pandemic.

$33 \%$ of participants reported that delays in diagnosis occurred very frequently.

17.2\% of participants reported that treatment abandonment occurred very frequently during the pandemic.

42.4\% of participants reported an increase in family out-of-pocket expenses

\section{Hosted file}

Table 1 PBC SLAOP COVID 19.docx available at https://authorea.com/users/457801/articles/ 554498-impact-of-covid-19-in-pediatric-oncology-care-in-latin-america-during-the-firstyear-of-the-pandemic 
Figure 1: Proportion of responses on childhood cancer treatments disruptions (total or partial suspensions) during the first year of COVID-19 pandemic in all participating Latin American countries.

\section{TREATMENT DISRUPTIONS PER SURVEY}

\section{LATIN AMERICA}

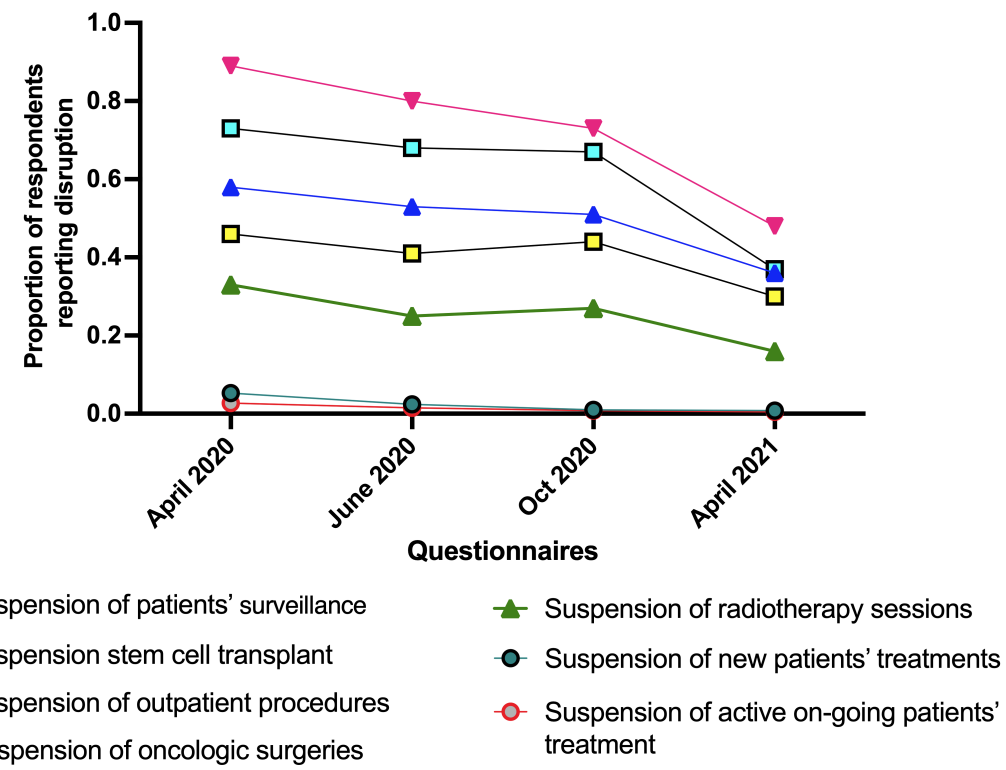


Figure 2: South America. Proportion of responses on treatments disruptions (total or partial suspensions) per survey and per country, compared to number of COVID-19 cases/day in each country

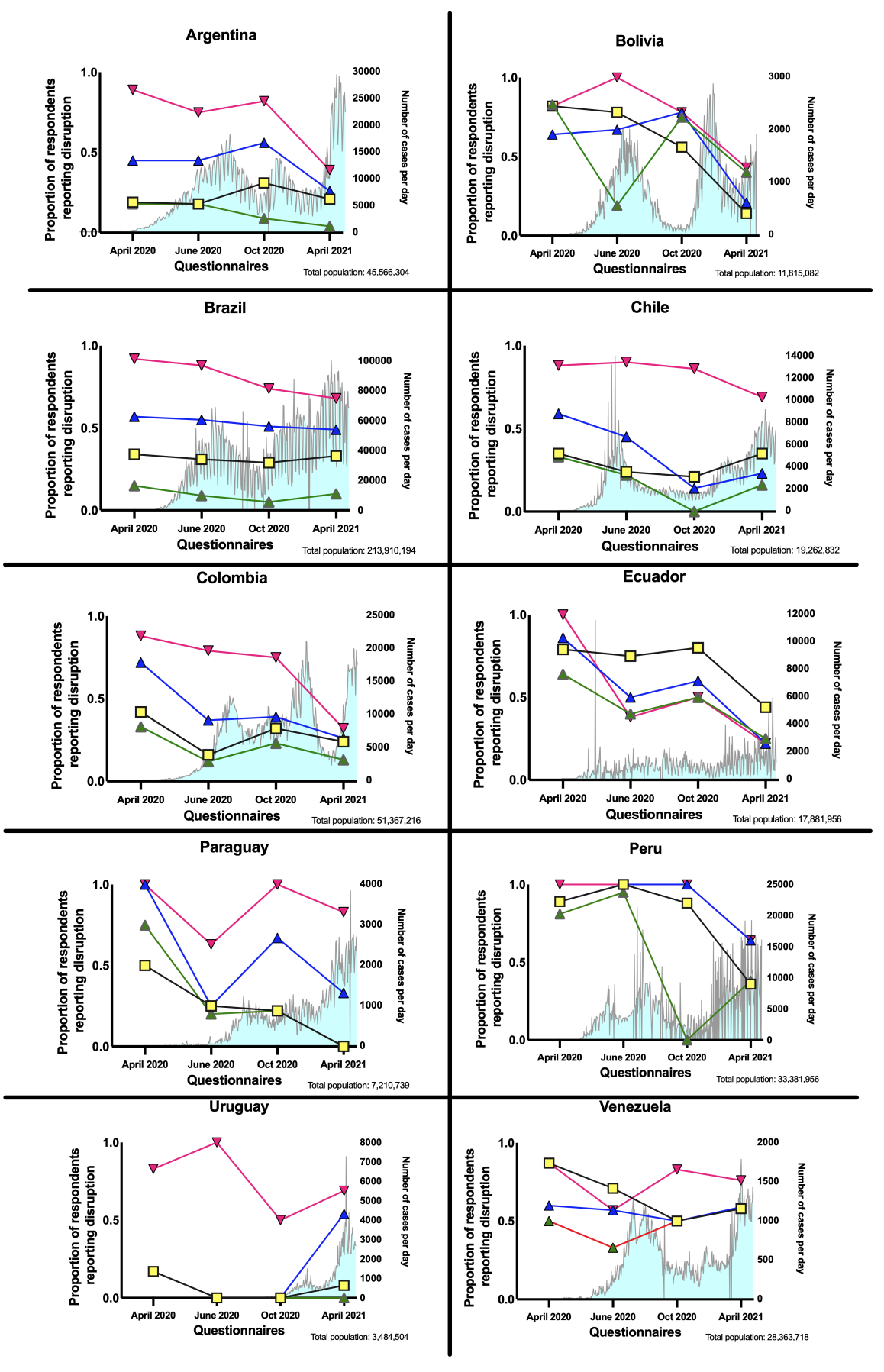


Figure 3: Central America. Proportion of responses on treatments disruptions (total or partial suspensions) per survey and per country, compared to number of COVID-19 cases/day in each country.
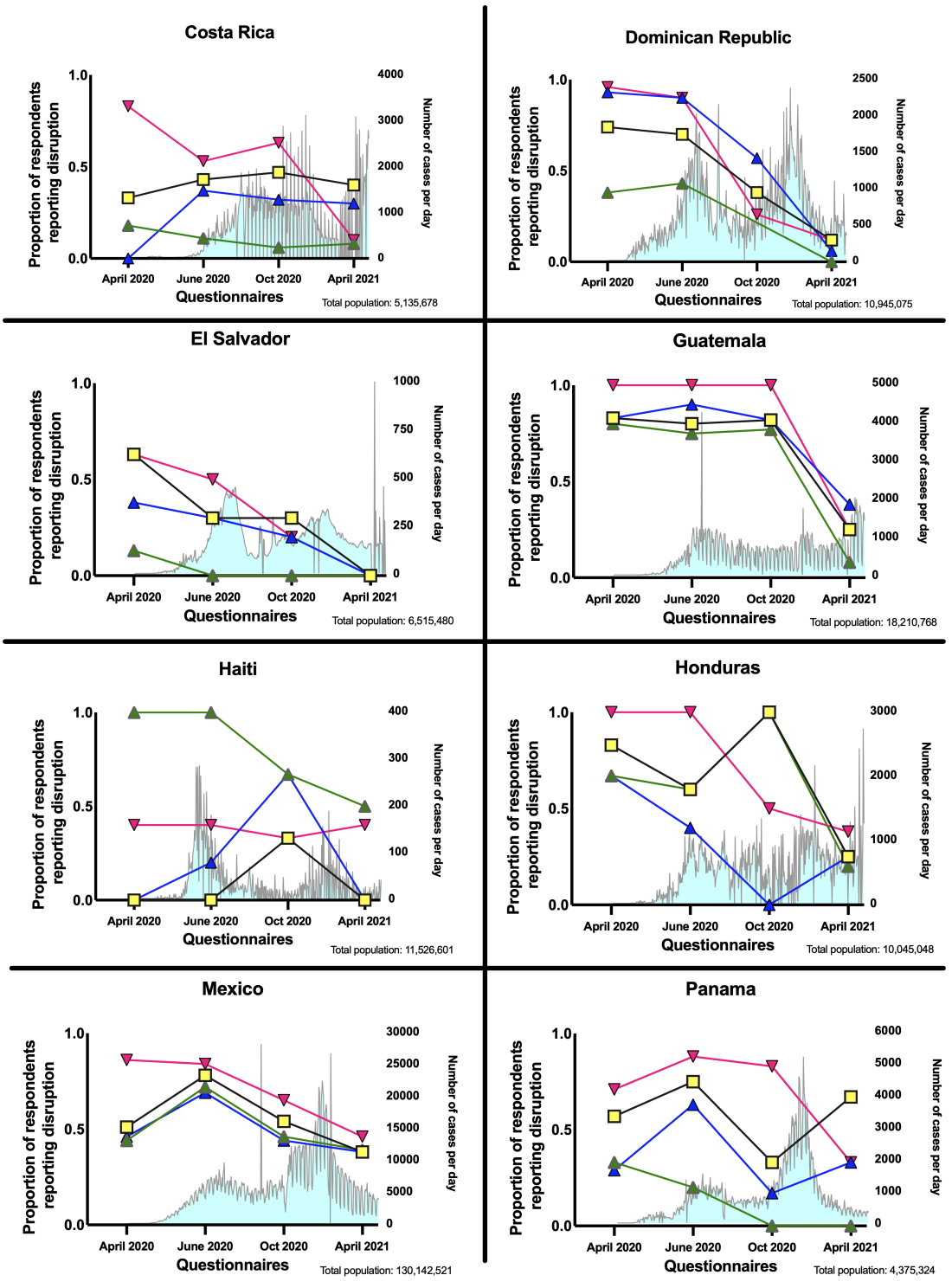

$$
\begin{aligned}
& \nabla \text { Suspension of patients' surveillance } \\
& \triangle \text { Suspension of outpatients' procedure } \\
& \square \text { Suspension of oncologic surgeries } \\
& \leftarrow \text { Suspension of radiotherapy }
\end{aligned}
$$


Figure 4: Effect of COVID-19 pandemic on treatment abandonment, delays in diagnosis and increase out-of-pocket expenses during the first year of the pandemic.

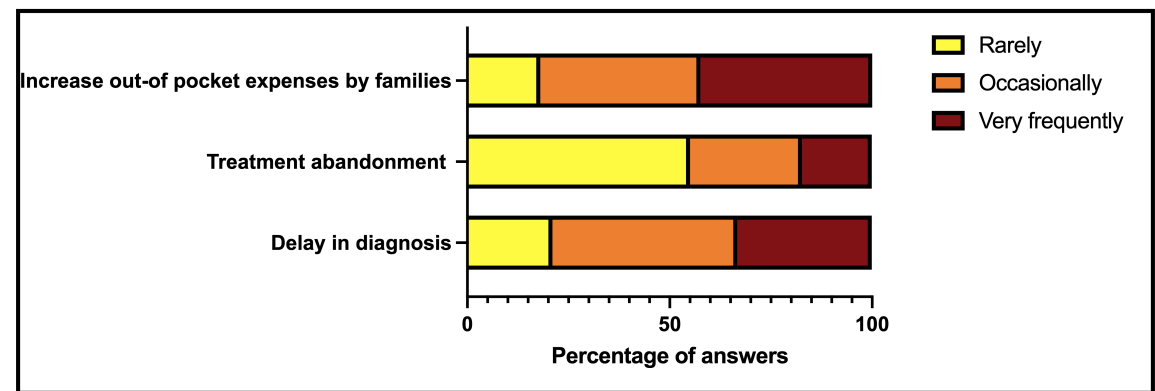

$33 \%$ of participants reported that delays in diagnosis occurred very frequently.

$17.2 \%$ of participants reported that treatment abandonment occurred very frequently during the pandemic.

$42.4 \%$ of participants reported an increase in family out-of-pocket expenses 